\title{
Delitos sexuales infanto-juveniles perpetrados por mujeres: caracterización y análisis fenomenológico en el contexto chileno*
}

\author{
Sexual Offenses Committed by Women against \\ Children and Adolescents: Characterization and \\ Phenomenological Analysis in the Chilean Context
}

Recibido: 9 de enero de 2013 | Revisado: 27 de marzo de 2014 | Aceptado: 24 de julio de 2014

\author{
Paula Denisse Flores ZúÑIGA ** \\ MARÍA ISABEL SALINAS **** \\ Universidad de Chile, Santiago, Chile
}

doi: 10.11144/Javeriana.upsy14-1.dsij

Para citar este artículo: Flores, P. D. (2015). Delitos sexuales infanto-juveniles perpetrados por mujeres: caracterización y análisis fenomenológico en el contexto chileno. Universitas Psychologica, 14(1), 137148. http://dx.doi.org/10.11144/Javeriana.upsy14-1. dsij

* Artículo original resultado de investigación

** Psicóloga, Estudiante Doctorado en Psicología, Universidad de Chile, Santiago, Chile.

**** Doctora en Psicología, Universidad de Chile, Santiago, Chile.

\section{RESUMEN}

Este artículo presenta una aproximación exploratorio-descriptiva a los delitos sexuales cometidos por agresoras mujeres contra niños, niñas y adolescentes. A partir de las características fenomenológicas de los delitos sexuales, se toman algunas de las principales variables identificadas, tales como vínculo víctima-victimaria, estrategia de sometimiento, tipo de delito, entre otras, las que se investigaron a través de un enfoque cuantitativo, teniendo como fuentes de información expedientes judiciales e informes psicológicos forenses. Los principales hallazgos indican que la mayoría de las víctimas infanto-juveniles son preescolares y tienen un vínculo de confianza y/o afecto con la agresora y la clase de contacto sexual impuesta por la autora está en relación con la edad de la víctima. A modo de conclusión, se aprecia un vuelco fenomenológico respecto a la casuística en agresores hombres, siendo el rol de cuidadora de la víctima el que cobra protagonismo. Palabras clave

agresoras sexuales; delitos sexuales; psicología forense

\footnotetext{
A B S T R A C T

This article is a phenomenological descriptive-exploratory approach to sex offenses committed by women against children and adolescents, both male and female. The study is based on the quantification of the main variables generally taken into account by the specialized literature, such as victimoffender bond, victim, victimization strategy, nature of the offense, among others. The sources of information used for the investigation consisted of legal records and psychological reports, both provided by an institution which has an assisting role to the Chilean legal system. The main findings indicate that most of the victims belong to nursery school and have a publically known bond to the offender. Also, the strategy used to achieve submission of the victim as well as the nature of the sexual contact itself are both likely to be related to the age of the victim. As a conclusion, a phenomenological turn can be recognized regarding the place of the offender in the victim's life, in which the role of care-taker becomes crucial in the comprehension of this kind of offence.

Keywords

female sex offenders; sex offenses; forensic psychology
} 


\section{Introducción}

A nivel empírico, aparece que en las agresiones sexuales a niños, niñas y adolescentes, la mayor frecuencia respecto al autor está asociada al hombre. Otra característica relevante es que la mayor incidencia se observa al incluir la variable vínculo entre víctima y victimario. No necesariamente se trata de vínculos intrafamiliares, sino también de nexos de confianza asociados al rol que cumple el victimario en la vida de la víctima (González, Martínez, Leyton, \& Bardi, 2004; Salinas, 2006).

El escaso interés en investigar a mujeres perpetradoras de delitos sexuales está asociado con aspectos culturales donde se contempla socialmente al hombre desde una posición de poder posible para cometer este tipo de ilícitos, no así otros, ya que se consideran igualmente responsables a hombres y a mujeres en lo que respecta a golpes, malos tratos u otros tipos de violencia contra niños y niñas (González et al., 2004).

Lo anterior resulta coherente con el abordaje histórico desde donde se ha descrito la criminalidad femenina: caracterizada como un fenómeno de menor impacto en comparación a la masculina, asociada a una tendencia marcada a determinados tipos de delitos en los cuales la mujer operaba generalmente sola o en complicidad con un hombre (Romero \& Aguilera, 2002), conllevando un escaso tratamiento empírico, donde las características de las ofensoras mujeres son delegadas como una consecuencia residual y/o excluida por su efecto perturbador dentro del tratamiento de datos en las investigaciones. En Chile, al igual que en países de habla hispana, la investigación de género y criminalidad es notoriamente escasa en comparación con países anglosajones, tal como señala Noemí (2004), en América Latina hasta la actualidad los estudios en esta área no pasan de ser un apéndice de los estudios criminológicos.

Particularmente, es en el espectro de los delitos sexuales donde la imagen de la mujer sustentada en percepciones basadas en estereotipos sobre la femineidad -vinculadas exclusivamente al rol materno y protector - resulta impensable asociar la condición de ser mujer con la criminalidad sexual (Abadi,
Beovide, \& Quattrone, 2001). Otro factor asociado a la escasa investigación y problematización sobre la incidencia es, tal vez, que se trata de un delito que atañe a la sexualidad, en donde históricamente la mujer ha jugado un papel pasivo. No obstante, resulta inevitable pensar que la criminalidad sexual masculina no refleja la casuística total, surgiendo el sexo del agresor como una variable crítica dentro de la fenomenología de este tipo de delitos.

Es importante señalar que la investigación en esta línea no ha estado exenta de críticas respecto a sus limitaciones metodológicas, frecuentemente asociadas al tamaño de las muestras (Christians \& Thyer, 2002 citados en Christopher, Lutz-Zois, \& Reinhardt, 2007), constitución de las mismas (Boroughs, 2004; Christopher et al., 2007; JohssonLove \& Fremouw, 2006) y el corte cualitativo de los estudios en la configuración de conclusiones.

En ese orden de ideas, en la Tabla 1 se presenta una síntesis de los principales hallazgos en la literatura empírica especializada, destacándose la descripción a partir de las características fenomenológicas esenciales.

Por otra parte, en relación con las características de las ofensoras sexuales, la literatura especializada señala que se caracterizan por ser mujeres jóvenes (Pereda, 2006), con una media de edad en el rango de los 26 a 36 años (Gannon \& Rose, 2008), por lo general vinculadas a trabajos asociados con la población infantil a nivel de roles estereotipados en la sociedad (Faller, 1987 citado en Gannon \& Rose, 2008; Pereda, 2006). Respecto al origen familiar de estas, se señala que provienen de familias disfuncionales y caóticas, donde frecuentemente han sido víctimas de malos tratos emocionales, físicos o sexuales (Boroughs, 2004; Pereda, 2006). En el ámbito social, se describen como mujeres aisladas (Pereda, 2006), que carecen de un sentido de pertenencia en relación con su grupo de pares, intentando a menudo buscar la aceptación de sus amigos de formas anormales (Matthews, $1993 \mathrm{ci}$ tado en Boroughs, 2004).

Sin embargo, esta información debe leerse con precaución, ya que no es posible establecer una causalidad lineal entre dichas características y la comisión de un delito sexual, eso sería un reduc- 


\section{TABLA 1}

Síntesis de hallazgos de la literatura especializada respecto las agresiones sexuales a niños, niñas y adolescentes, perpetradas por mujeres

\begin{tabular}{|c|c|}
\hline $\begin{array}{l}\text { Edad de la } \\
\text { víctima }\end{array}$ & $\begin{array}{l}\text { Prepúberes (Faller, 1987; Vandiver \& Walker, } 2002 \text { citados en Gannon \& Rose, 2008). } \\
\text { Adolescentes entre } 12 \text { y } 17 \text { años (Sandler \& Freeman, 2007; Vandiver \& Kercher, } 2004 \text { citados en Bader, } \\
\text { Scalora, Casady, \& Black, 2008). }\end{array}$ \\
\hline $\begin{array}{l}\text { Sexo de la } \\
\text { víctima }\end{array}$ & $\begin{array}{l}\text { Masculino } \\
\text { Son más propensos a ser agredidos sexualmente por mujeres, especialmente en la etapa de su adolescencia } \\
\text { (Finkelhor \& Russell, 1984; Fromuth \& Conn, } 1997 \text { en Gannon \& Rose, 2008). } \\
\text { Presente mayoritariamente en casos incluidos en muestras de víctimas que ingresan a la justicia penal (Bader } \\
\text { et al., 2008). } \\
\text { Femenino } \\
\text { Se encuentra principalmente asociado a aquellos casos no denunciados a la justicia (Bader et al., 2008). } \\
\text { Ambos géneros/Sin preferencia } \\
\text { No tienen un sexo de víctima preferida (Becker et al 2001; Grayston \& De Luca, 1999; Love-Johansson \& } \\
\text { Fremouw, 2006 citados en Johansson-Love \& Fremouw, 2009). }\end{array}$ \\
\hline $\begin{array}{l}\text { Vínculo } \\
\text { víctima-agresora }\end{array}$ & $\begin{array}{l}\text { Conocida } \\
\text { En la gran mayoría de los casos, la víctima conoce a la agresora; está relacionado con el lugar que ocupa la } \\
\text { agresora en el entorno cercano de la víctima (Correctional Service Canada, 2008; Gannon \& Rose, 2008). } \\
\text { Kaufman, Wallace, Johnson y Reeder (1995) encontraron que más del 75\% de las víctimas son agredidas por } \\
\text { mujeres que están relacionadas con ellas (Bader et al., 2008). } \\
\text { Intrafamiliar } \\
\text { Johansson-Love y Fremouw (2009) sugieren que los delincuentes sexuales femeninos (58\%) están más } \\
\text { propensos que los varones (23\%) a estar biológicamente relacionados con su víctima. } \\
\text { Muchas de las víctimas tienden a ser los propios hijos del delincuente (Correctional Service Canada, 2008). } \\
\text { En particular, en la muestra del Centro de Protección Infantil había víctimas mayoritariamente de vínculo } \\
\text { intrafamiliar (97.8\%) (Bader et al., 2008). } \\
\text { Desconocida } \\
\text { No hay hallazgos en la literatura especializada que den cuenta de casos de mujeres ofensoras que } \\
\text { desconozcan a su víctima (Beech, Parrett, Ward, \& Fisher, 2009). }\end{array}$ \\
\hline $\begin{array}{l}\text { Forma } \\
\text { sometir }\end{array}$ & $\begin{array}{l}\text { Uso de fuerza física } \\
\text { Mathews et al., } 1997 \text { en Gannon y Rose, informaron que no hubo diferencias en el uso de la fuerza física } \\
\text { durante la agresión sexual, entre el grupo de agresores varones y el de agresoras mujeres (Gannon \& Rose, } \\
\text { 2008). } \\
\text { Victimización junto a un coautor } \\
\text { Kaufman et al., } 2005 \text { en Beech et al. encontraron que las mujeres delincuentes sexuales tienen más } \\
\text { probabilidades de participar con un coautor (Beech et al., 2009). } \\
\text { Uso de sustancias, drogas y/o alcohol en sus víctimas } \\
3.9 \% \text { de las agresoras sexuales incluidas en la muestra del estudio (Bader et al., 2008). } \\
\text { Utilización de armas } \\
\text { No hay registro en los casos incluidos en estudios empíricos ni en el desarrollo teórico del tema. }\end{array}$ \\
\hline $\begin{array}{l}\text { Clase de } \\
\text { contacto sexual }\end{array}$ & $\begin{array}{l}\text { No existe registro en la literatura especializada sobre un desarrollo acabado respecto al tipo de agresión } \\
\text { sexual. } \\
\text { Tardif, Auclair, Jacob y Carpentier (2005) dan cuenta del espectro de las conductas sexuales invasivas } \\
\text { detectadas en su estudio. De manera descendente según frecuencia, se encuentran: caricias sexuales a su } \\
\text { víctima, masturbación a la víctima, incitar a que la víctima las masturbara, felación en frente de la víctima, } \\
\text { contacto oral-genital con la víctima, contacto oral-genital por la víctima, penetración digital a la vagina, } \\
\text { coito con su víctima, entre otras. }\end{array}$ \\
\hline Frecuencia & $\begin{array}{l}\text { En el estudio de Tardif et al. (2005), las agresiones se consignaron como; solo algunos eventos (23.1\%), entre } \\
10-35 \text { eventos (38.5\%) y un par de veces a la semana (23.1\%). } \\
\text { Dependiendo del tipo de agresión sexual y el vínculo agresora-víctima, la mayoría de las víctimas devela } \\
\text { agresiones sexuales que se prolongan desde seis meses a seis años (Denov, 2002; Tardif et al., 2005). }\end{array}$ \\
\hline $\begin{array}{l}\text { Daño } \\
\text { psicológico } \\
\text { asociado }\end{array}$ & $\begin{array}{l}\text { Vínculo conocido } \\
\text { Sentimientos de traición y rabia (Boroughs, 2004). } \\
\text { Saradjian y Hanks, } 1996 \text { citados en Borough (2004) señalan que las víctimas se autoperciben mayormente } \\
\text { estigmatizadas, traicionadas e impotentes, en comparación a las víctimas de agresiones sexuales perpetradas } \\
\text { por hombres. Los mismos autores señalan que dichas víctimas al entrar en la edad adulta presentarían } \\
\text { incapacidad para mantener relaciones interpersonales sanas. } \\
\text { Vínculo intrafamiliar } \\
\text { En Kelly, Wood, Gonzalez, MacDonald y Waterman (2002), se da cuenta de que los hombres agredidos } \\
\text { sexualmente por autoras maternas presentaban mayor grado de disturbios en diversas áreas del } \\
\text { funcionamiento psicosocial que aquellos que fueron agredidos por agresoras conocidas. Estos resultados, que } \\
\text { coinciden con Mendel (1995) en Strickland (2008), se suman a la literatura empírica muy limitada sobre los } \\
\text { efectos a largo plazo del incesto madre-hijo. }\end{array}$ \\
\hline
\end{tabular}

Fuente: elaboración propia 
cionismo lógico. Lo que sí es posible deducir es que los perfilamientos criminales por sexo son diferentes en algunas características fundamentales, que permitirían hacer distinciones significativas aplicables tanto al tratamiento de las víctimas y victimario/a como también a la prevención general (Saradjian \& Hanks, 1996 citados en Robertiello \& Terry, 2007).

En virtud de los antecedentes, la presente investigación da cuenta de una temática escasamente investigada en contexto latinoamericano, lo que se constituye en un aporte al conocimiento. Se pregunta por icuáles son las características de los delitos sexuales infanto-juveniles perpetrados por mujeres, ingresados al sistema judicial en un proceso investigativo entre los años 2007 y 2010 en Chile?

De esa forma, a través de un acercamiento exploratorio se contempló como objetivos identificar y definir las principales variables utilizadas en la caracterización de los casos de delitos sexuales infanto-juveniles perpetrados por mujeres - a partir de la revisión de la literatura empírica especializada y la opinión de expertos chilenos en la materia- y en relación con estas, describir los delitos sexuales en contra de niños, niñas y adolescentes perpetrados por mujeres en Chile, para de ese modo sistematizar los principales hallazgos en la muestra y lo expuesto por la literatura especializada.

\section{Método}

\section{Procedimiento}

Para dar respuesta a los objetivos de investigación, se optó por un diseño no experimental, transeccional y descriptivo, bajo un enfoque cuantitativo, constituyéndose en una investigación de tipo exploratorio y descriptivo.

La recolección de información se realizó a través de dos fuentes secundarias de información: la carpeta investigativa o Expediente Judicial y el Informe Psicológico Pericial, datos proporcionados por la Corporación OPCIÓN, que tiene a su cargo 17 Programas de Diagnóstico Ambulatorio en Chile.

\section{Población y muestra}

La población considerada en esta investigación son todos los casos de delitos sexuales de víctimas menores de 18 años, donde se establezca la denuncia en contra de un imputado de sexo femenino, entre los años 2007-2010 en Chile. Al no existir un registro estadístico que considere la variable sexo del imputado en esta clase de delitos denunciados en Chile, se estimó el tamaño poblacional a través del criterio teórico de la prevalencia internacional del fenómeno (cercana al 4\%), el cual fue identificado en el promedio de casos ingresados (290 casos) al Servicio $\mathrm{Na}$ cional de Menores de Chile por vulneración de derechos en el área de agresiones sexuales entre los años 2007 y 2010.

La composición de la muestra fue de 20 casos, seleccionada a partir de un muestreo no probabilístico, debido a que se escogió a partir de un procedimiento de selección dirigida, incluyéndose todos aquellos casos de delitos sexuales infantojuveniles perpetrados por una mujer, constando como imputada en la investigación emprendida por el Ministerio Público.

\section{Definición de variables}

Las variables que se consideraron en este estudio fueron extraídas de los hallazgos de las investigaciones especializadas reseñadas. Fueron definidas a partir de los aportes de la investigación de Salinas (2006), donde se levantaron descripciones fenomenológicas del análisis de 100 casos de víctimas de delitos sexuales. Además, cada una de estas variables, así como sus definiciones conceptuales y operacionales, fue validada por un grupo de tres jueces expertos en evaluación forense en delitos sexuales, con el fin de asegurar su correcta utilización y relevancia en el medio chileno. Entre las variables consideradas en la investigación, se contempló el sexo y edad de la víctima y aquellas relacionadas con las características del hecho denunciado, forma de la agresión sexual y daño psicológico asociado (Tabla 2). 
Tabla 2

Definiciones de las variables a nivel conceptual y operacional de la investigación

\begin{tabular}{ll}
\hline Variable & Definición Conceptual \\
\hline $\begin{array}{l}\text { Características } \\
\text { de la denuncia }\end{array}$ & $\begin{array}{l}\text { Denunciante } \\
\text { Identificación de la persona que realiza la } \\
\text { denuncia sobre los hechos que se investigan. }\end{array}$
\end{tabular}

Definición Operacional

Persona intrafamiliar: Persona con vínculo sanguíneo

o sin vínculo sanguíneo de la víctima del ilícito, que

cumple un rol en su grupo familiar.

Persona conocida: Persona que ocupa un lugar en la vida de la víctima, ya sea por cercanía física, rol o por afecto.

La propia víctima: Persona afectada por los hechos que se investigan.

Institución: Profesional o persona natural vinculada a una institución social, salud, educacional u otra, que enterada del presunto delito establece la denuncia.

Delito denunciado

Delito dispuesto en el Código Penal Chileno, Libro II, Título VII de los crímenes y delitos contra el orden de las familias, contra la moralidad pública y contra la integridad sexual.

Vínculo víctima- Relación existente entre la autora del delito y agresora la víctima al momento de la ocurrencia de los hechos abusivos.
El delito denunciado que se encuentre presente en el Parte de Denuncia ante Carabineros de Chile o Fiscalía, correspondiente al caso.

Intrafamiliar: Relación mediada por un vínculo de parentesco familiar (sanguíneo/no sanguíneo) entre la autora del delito y su víctima.

Conocido: Relación dada por la posición que ocupa la autora en la vida de la víctima, siendo mediada por la cercanía física, el rol y/o el afecto.

Desconocido: Relación entre la autora y su víctima bajo ningún tipo de vínculo.

Forma de Forma de sometimiento empleada por la autora sometimiento

Fuerza física: Utilización de violencia física por parte de la autora hacia la víctima. para cometer el delito sexual. Cabe señalar, que las categorías no son excluyentes, pudiendo presentarse más de alguna.
Mediante uso de armas: Utilización de cualquier tipo de arma empleada por la autora para propiciar el sometimiento de la víctima.

Amenazas personales: La autora da entender con actos o palabras que de no acceder al requerimiento puede causar algún daño a la víctima.

Amenazas a terceros: La autora da entender con actos o palabras que de no acceder al requerimiento puede causar algún daño a un tercero significativo en la vida de la víctima.

Abuso de poder: Proviene del rol que ocupa la autora en la vida cotidiana de la víctima.

Manipulación a través de la seducción y/o afecto: Aproximaciones sucesivas tendientes a generar un vínculo erótico por parte de la autora. El delito denunciado que se encuentre presente en el Parte de Denuncia ante Carabineros de Chile o Fiscalía, correspondiente al caso.

\begin{tabular}{ll}
\hline Clase contacto & Clase de contacto corporal o no corporal de \\
sexual & connotación sexual, establecido entre la autora \\
& y su víctima
\end{tabular}

Caricias de connotación sexual de la autora al cuerpo de la víctima.

Masturbación de la autora a la víctima, implicando necesariamente el contacto con la zona genital de su víctima.

Masturbación de la propia autora frente a la víctima. Contacto oral-genital con la víctima por la ofensora. Contacto oral-genital por la víctima a la autora. Introducción de objetos anal/vaginal a la víctima por la autora.

Penetración digital anal/vaginal a la víctima por parte de la autora.

Coito con su víctima por parte de la autora.

Otro tipo de conducta sexual.

\begin{tabular}{lll}
\hline Frecuencia & Número de episodios en que la autora efectúa & Episodio único: Solo un episodio abusivo por parte de \\
& $\begin{array}{l}\text { las conductas de connotación sexual a su } \\
\text { víctima. }\end{array}$ & $\begin{array}{l}\text { la autora. } \\
\text { Reiterado: Más de un episodio de carácter abusivo por } \\
\text { parte de la autora. }\end{array}$ \\
\end{tabular}




\begin{tabular}{lll}
\hline Develación & $\begin{array}{l}\text { Tipo de develación } \\
\text { La ubicación temporal de la develación } \\
\text { respecto de la ocurrencia del delito. }\end{array}$ & $\begin{array}{l}\text { Reactiva: Inmediata a la ocurrencia del primer } \\
\text { acontecimiento abusivo de carácter sexual. } \\
\text { Tardía: Posterior a la ocurrencia de los acontecimientos } \\
\text { abusivos de carácter sexual. }\end{array}$ \\
\hline & $\begin{array}{l}\text { Forma de develación } \\
\text { Manera en que se toma conocimiento el hecho } \\
\text { que se debe investigar. }\end{array}$ & $\begin{array}{l}\text { Directa: La víctima devela intencionalmente a una } \\
\text { figura directa de apoyo, por ejemplo, al padre o la madre. } \\
\text { Indirecta: Devela intencionalmente a una figura } \\
\text { indirecta de apoyo, por ejemplo, la profesora. }\end{array}$ \\
& $\begin{array}{l}\text { Circunstancial: La víctima no tiene intención de } \\
\text { develar; el entorno se percata por indicios externos } \\
\text { relacionados con la ocurrencia de los hechos abusivos. }\end{array}$ \\
\hline $\begin{array}{l}\text { Daño psicológico } \\
\text { explícito }\end{array}$ & $\begin{array}{l}\text { Cualquier interferencia significativa, psíquica } \\
\text { o social, identificada por el perito en el informe } \\
\text { pericial psicológico, que altere el continuo vital } \\
\text { del desarrollo del niño, niña o adolescente. }\end{array}$ & $\begin{array}{l}\text { Presencia de daño psicológico explícito en la víctima. } \\
\text { Ausencia de daño psicológico explícito en la víctima. }\end{array}$ \\
& & \\
\hline
\end{tabular}

Fuente: elaboración propia

\section{Análisis de datos}

El análisis de los datos se hizo a partir de la calificación de las características de los casos de la muestra, en función de las variables anteriormente definidas. En cada caso, se sistematizó la información de los instrumentos a través de una ficha de registro de análisis, que en la parte inicial incluyó la identificación del caso junto a una breve reseña respecto a los antecedentes relevantes, y posteriormente la cuantificación de cada una de las variables del estudio.

Considerando el tamaño de la muestra, se utilizaron técnicas propias del análisis estadístico descriptivo, expresando los resultados principalmente en porcentajes y utilizando a su vez algunas medidas de resumen como tablas de frecuencias y gráficos para su representación.

\section{Resultados}

Características de las víctimas de agresiones sexuales perpetradas por mujeres

La muestra estuvo compuesta por 20 víctimas de edades entre los 3 y 17 años, con una moda de 5 años, media 6.1 años y desviación estándar de 4.16 años. Del total, 9 correspondieron a mujeres y 11 a hombres, siendo la cantidad de hombres $15 \%$ superior al número de mujeres. Asimismo, respecto del nivel educacional de los sujetos, el 63.2\% de los casos se concentró en niños y niñas preescolares que aún no ingresan al sistema de educación formal.
En particular, la edad de las víctimas incluidas en la muestra al inicio de las agresiones sexuales se ubicó entre los 2 y 12 años, con una moda de 5 y 3 años, una media de 5.25 años y una desviación estándar de 3.27, concentrándose un $75 \%$ en edad preescolar.

Referente a las características de la denuncia, la persona que la interpuso, en el $75 \%$ de los casos (15 casos) se concentró en personas de vínculo intrafamiliar y $25 \%$ (5 casos) por personas miembros de alguna institución relacionada con la víctima, tales como jardín infantil, colegio, entre otros. De los delitos denunciados, el de abuso sexual a menor de 14 años contó con la mayor frecuencia (65\%). Cabe señalar que se incluyen dos casos donde la denuncia es establecida por "otros hechos que no constituyen delito", no obstante, al iniciarse la investigación son recalificados uno por abuso sexual a menor de 14 años y el otro por violación a mayor de 14 años (al momento de recabar el dato, la calificación del delito fue corregida por la entidad pertinente por no corresponder al tipo penal).

En relación con el vínculo entre la agresora y la víctima, el $40 \%$ de los casos correspondió a intrafamiliar asociado exclusivamente a la figura de la madre y un $60 \%$ a vínculo conocido. Las formas de sometimiento más frecuente fueron la de amenazas personales mostrada en 15 casos, seguida por la manipulación a través de la seducción o afecto (11) y abuso de poder (8). Es importante destacar que solo en un caso se presentó el uso de fuerza física. Cabe señalar la existencia de más de una forma de sometimiento por parte de la agresora sexual a su víctima en un mismo caso. 
Las clases de contacto sexual más frecuentes se encontraron en el tipo de caricias de connotación sexual de la autora al cuerpo de la víctima (30\%), contacto oral-genital con la víctima (16.66\%), seguidas en una misma proporción (10\%) por penetración digital a la vagina de la víctima, introducción de objetos anal/vaginal y coito con la víctima. Por cierto, cada caso presentó más de un tipo de contacto, no siendo excluyentes entre sí.

En cuanto a la frecuencia de las agresiones sexuales en la muestra, un $70 \%$ revistió carácter reiterado y un 30\% episodio único. En cuanto al tipo y forma de develación, en 15 de los casos las víctimas no presentaron intención o voluntad por develar los hechos, lo que implica que no se obtienen datos sobre la data de inicio de las agresiones sexuales, categorizándose por tanto como circunstancial (75\%). De las develaciones restantes, en 4 de los casos, fueron de tipo directa-tardía y en un caso develación indirecta-reactiva a la ocurrencia del delito denunciado.

Cabe señalar que en relación con la exposición cuantitativa de los datos de la variable de daño explicito, en el diseño se estableció como indicador operacional la sintomatología física, emocional, relacional o de desajuste a la vida cotidiana, en todos los contextos de interacción de las unidades muestrales, pero no fue posible levantar datos debido a la ausencia de criterios explícitos para medir dicha variable. Solo se registró en razón de presencia y ausencia, según lo indicado en la carpeta investigativa como el daño explícito identificado, resultando presente en el $65 \%$ de los casos y ausente en el 10\% de los casos. Es importante indicar que en cinco casos no se realizó la evaluación de daño, por lo tanto no hay ninguna referencia al respecto.

\section{Características de las agresoras sexuales}

Las ofensoras sexuales, todas de nacionalidad chilena exceptuando a una extranjera, se encuentran en un rango etario entre los 15 y 64 años de edad, con una moda de 30 años, una media de 30.6 años de edad y una desviación estándar de 11.75. En cinco de las imputadas, la edad es un dato desconocido, toda vez que no es consignado en la carpeta inves- tigativa. Respecto al estado civil de las imputadas -se dispuso de tal información en solo 12 de los casos- la mayoría era soltera (45\%).

En referencia al nivel educacional de las imputadas, el 15\% contaba con estudios medios completos y el $55 \%$ con estudios superiores, incluyendo universitarios o técnicos. Asimismo, un alto porcentaje tenía oficios o profesiones afines con el de "cuidadora” de niños y niñas en edad preescolar (45\%).

Respecto al número de víctimas asociadas a la misma imputada, en seis casos se presentó más de una víctima por imputada y en 12 casos solo una. En el resto de los casos, se desconoce este dato. Además se hace constar que 16 de las agresoras sexuales actuaron solas el cometer el delito (80\%), tres de ellas junto a otras personas (15\%) y una junto a su pareja (5\%).

\section{Análisis de datos}

Se analizaron y contrastaron las variables identificadas empíricamente en la literatura con los resultados de la muestra. Es importante señalar que toda observación fue de carácter hipótetico y exploratorio, teniendo en cuenta las limitaciones metodológicas que implica el abordaje del presente objeto de estudio.

En cuanto a la edad de inicio de las agresiones sexuales, existe un número mayor de víctimas vulneradas en edades inferiores a los 6 años, con una media de 5.25 años. Dicho antecedente, resulta similar a lo señalado por Onetto y Araya (2007), quienes indican que el porcentaje de víctimas en cada tramo etario va disminuyendo a medida que aumenta su edad; no obstante, en ese estudio la mayoría de las víctimas se concentró bajo un tramo de edad más amplio: los 10 años.

Al observar en conjunto el sexo de las víctimas por rangos de edad con el inicio de las agresiones sexuales, se aprecia que en las menores de 7 años no existen diferencias respecto al sexo, siendo indistintamente niños o niñas. Sin embargo, en el rango que supera los 12 años, se encuentran exclusivamente víctimas de sexo masculino lo que indica similitud con las agresiones sexuales infantiles perpetradas por hombres, en términos de heteroagresión. 
La supremacía de la cifra de develaciones de carácter circunstancial permite hipotetizar que las agresiones sexuales cometidas por mujeres se relacionan con una forma de sometimiento naturalizada a través de la socialización y que utiliza al rol de la agresora en la vida cotidiana de la víctima como un poderoso obstáculo que interfiere en la develación del delito y que a su vez contribuye a la perpetuación de una sintomatología vinculada a una interferencia en el desarrollo psicosexual de las víctimas.

Respecto a los dos delitos que inicialmente son denunciados como "Otros hechos que no constituyen delito" y que son recalificados al iniciar la investigación, es posible presumir que al momento de la denuncia no fueran considerados como un delito sexual por tratarse de hechos inusuales que tenían como protagonista a una mujer imputada. Lo anterior refuerza la hipótesis de la existencia de un sesgo de parte de los actores del sistema, que colaboraría con el mantenimiento de la cifra negra asociada a los delitos sexuales infantiles perpetrados por mujeres (Pereda, 2006). En ese mismo orden de ideas, los delitos sexuales cometidos por mujeres presentarían una dinámica específica que se nutre de una interacción que requiere un contexto de ocurrencia compatible con la cercanía física y afectiva. De este modo, en el rol que juega la agresora en la vida de la víctima, se encontraría el origen del despliegue de conductas de cuidado donde se camufla la forma de sometimiento de la agresora.

Al contrastar los resultados antes señalados con lo descrito en la literatura especializada, es congruente establecer el antecedente que remite a la menor utilización de fuerza física como forma de sometimiento por parte de la agresora comparada con los agresores masculinos (Gannon \& Rose, 2008; González et al., 2004; Pereda, 2006), así como la no utilización de armas (Bader et al., 2008). No obstante, existe discrepancia con los resultados del estudio de González et al. (2004) que plantean que las agresoras tienden a utilizar con menos frecuencia amenazas como forma de mantener el silenciamiento en su víctima. Ese dato puede estar asociado a una apreciación de la cualidad de la amenaza, siendo probable que esté siendo entendida como violenta y explícita y no como parte de la dinámica abusiva, donde, a partir de la instrumentalización del rol, se desfigura el involucramiento subjetivo de la víctima. En otras palabras, con mayor probabilidad las amenazas pueden ser frecuentes si se implican como parte esencial de la interacción abusiva y no solo como un medio de sometimiento.

En la literatura especializada al igual que en la presente investigación, las caricias de connotación sexual son las más frecuentes (Tardif et al., 2005). Del análisis en forma conjunta de las clases de contacto sexual con la edad de las víctimas, en el tramo de edad entre uno y tres años, fueron sometidas a un contacto sexual unidireccional e invasivo, tendiente a una visión cosificada de ellas por parte de las autoras, mientras que en las víctimas de cuatro a cinco años, las clases de contacto abusivo se remiten a la genitalidad de los niñas y niños, destacándose en ambos sexos acciones penetrativas por parte de la autora a través de objetos o digitalmente. En contraste a lo cotejado en víctimas preescolares, las agresoras desarrollaron conductas dirigidas a la bidireccionalidad con los adolescentes, que conllevaban una mayor combinación de clases de contacto.

En relación con la frecuencia de la dinámica abusiva, el 70\% fue de carácter reiterado y un 30\% episodio único. Al respecto, este último porcentaje es problemático ya que está mediado por la relación entre víctima y agresora; al analizarlo en el contexto de la dinámica abusiva, debería ser menor. La relación víctima-victimaria interfiere en la develación de los hechos abusivos, y por tanto impacta en la frecuencia, variable que tiene importancia legal en tanto funciona como agravante para establecer la pena.

\section{Discusión}

El reconocimiento del dato que alude a que la mayoría de los agresores sexuales infantiles tienen un vínculo con la víctima permite desmitificar la creencia de que en lo desconocido está el peligro. Aun cuando se trate de un victimario hombre o mujer, la criminalidad sexual apunta poderosamente a sostener, como parte de su dinámica, el abuso de confianza y/o afecto. 
Es obvio que la utilización de la fuerza es necesaria o es más lógico usarla cuando se trata de una víctima desconocida, no así en las que existe un vínculo previo de confianza y/o afecto entre la pareja penal. Si la mayor frecuencia se observa en esta última categoría, los resultados muestran que no existen diferencias según sea el sexo del victimario; sin embargo, se observan diferencias en la cualidad de la acción abusiva. En este estado de situación, el problema tiene dos aristas que se influencian mutuamente: la primera refiere a la tendencia cultural androcentrista que obstaculiza la condición de posibilidad de prevenir o bien actuar de manera reactiva frente al delito y la segunda, a la dinámica abusiva realizada por la victimaria que tiende a ser naturalizada tanto por el contexto como por la misma víctima. Sin duda, que la naturalización está basada en la construcción social de los roles de cuidado y, tal como se presentó en esta investigación, la estrategia de sometimiento de la victimaria está sustentada sobre un rol de cuidado vinculado al género. Por lo tanto, es ese rol el que está en la base de la incredulidad del contexto y refuerza el secreto.

Históricamente, se ha problematizado la violencia sexual desde una perspectiva criminal masculina, marginándose el análisis de las características de la ofensora sexual y su modus operandi según sea el delito. Los resultados de la presente investigación, así como los expuestos por la literatura especializada anglosajona, hacen referencia a que la criminalidad sexual femenina se comporta en forma similar a la de los ofensores hombres en solo algunas variables, específicamente en relación con la elección de la víctima: el rango etario bajo los 6-7 años, la elección es indiscriminada por sexo, pero luego el sexo femenino en el caso de los agresores hombres obtiene una mayor frecuencia. Un aspecto del análisis sobre la elección del sexo de la víctima en la criminalidad sexual masculina es que esta no se relaciona directamente con la orientación y/o atracción sexual del ofensor sexual, dado que en un porcentaje importante este se caracteriza como heterosexual. En el caso de la mujer criminal no existen antecedentes empíricos que permitan reflexionar sobre aquellos ca- sos donde la agresión se dirige hacia una víctima del mismo sexo, lo que deja abierta una línea de investigación interesante.

Asimismo, estos hallazgos generan nuevas necesidades de conocimiento, por ejemplo, sobre un concepto gravitante en la victimización sexual: el constructo de daño. Los datos arrojados por esta investigación dirigen el análisis a problematizar sobre el daño ocasionado, vinculado a la victimización primaria. Las víctimas estudiadas presentaban un mayor porcentaje de develaciones circunstanciales, es decir que no existe una voluntad de solicitar ayuda externa. Dicho dato resulta completamente diferente de la fenomenología de las agresiones sexuales cometidas por hombres, donde un porcentaje menor corresponde a esta categoría (Salinas, 2006). Se entiende que parte de la cifra negra del delito sexual se relaciona con el tipo y forma de develación, es decir, esa información es un predictor de las demandas de ayuda de la víctima que no han sido proporcionadas por los terceros a los que esta accede. Pero el problema reviste mayor dificultad, si la víctima no exhibe motivación para esta demanda. Los factores en la base de la ausencia de voluntad pueden estar vinculados a un contexto sociorreferencial -no tener vínculos significativos- o bien, a factores subjetivos -desconocimiento de su rol como víctima al interior de la dinámica abusiva-. Sin duda, ambos factores son generadores y/o potenciadores de daño psíquico, eso sí, con procesos y construcciones distintas.

Retomando los hallazgos, es posible problematizar la articulación entre las condiciones situacionales, la forma de sometimiento, el vínculo de poder que otorga el ejercicio de un rol en la vida de la víctima, el contexto de ocurrencia en una cultura androcentrista y el daño psicológico en las víctimas. Este último podría tener incidencias significativas en el desarrollo psicosexual de las víctimas, argumento sustentado en las concepciones de género imperante en la sociedad patriarcal, ya que desde ahí se propicia el surgimiento de sentimientos de confusión y traición, impactando negativamente la identificación o lectura de las conductas abusivas y, por tanto, el posicionamiento de la víctima dentro de la relación abusiva. 
En virtud de lo anterior, resulta relevante la evaluación de los diagnósticos e impacto de daño psicológico en este tipo de víctimas. Es decir, es necesario reconocer que la variable de género resulta crucial para la construcción del diagnóstico del daño, debiendo contemplarse indicadores operacionales específicos para este tipo de casos. Dicha visión tiene un impacto directo en las estrategias de prevención de posibles eventos abusivos asociados a conductas criminales encubiertas por las representaciones sociales de la mujer y contribuyendo a la persistencia de la cifra negra del fenómeno.

Las implicancias del presente estudio se relacionan con el reconocimiento de un fenómeno que parece ser relegado a la incredulidad, dando cuenta a través de un acercamiento exploratorio, que este no parece tan infrecuente como se esperaría. Con el objetivo de aumentar la comprensión de la criminalidad sexual femenina, como también el conocimiento sobre las víctimas y consecuencias asociadas a estas, se sugieren investigaciones futuras, en las cuales no tan solo se realice un abordaje del sexo del victimario/a en forma nominal (como por ejemplo en el registro de encuestas de victimización o bases de antecedentes judiciales), sino que la investigación se oriente al tratamiento del género del agresor/a sexual, considerando aspectos socioculturales.

\section{Referencias}

Abadi, G., Beovide, M., \& Quattrone, A. (2001). La invisibilidad de la violencia sexual en el vínculo de las madres con sus hijos/as. Buenos Aires: Ediciones C.S.M.

Bader, S., Scalora, M., Casady, T., \& Black, S. (2008). Female sexual abuse and criminal justice intervention: A comparison of child protective service and criminal justice samples. Child Abuse $\mathbb{E}$ Neglect, 32, 111-119.

Beech, A., Parrett, N., Ward, T., \& Fisher, D. (2009). Assessing female sexual offenders' motivations and cognitions: An exploratory study. Psychology, Crime E Law, 15(2-3), 201-216.

Boroughs, D. (2004). Female sexual abusers of children. Children $\mathfrak{E}$ Youth Services Review, 26(5), 481-487.
Christopher, K., Lutz-Zois, C., \& Reinhardt, A. (2007). Female sexual-offenders: Personality pathology as a mediator of the relationship between childhood sexual abuse history and sexual abuse perpetration against others. Child Abuse $\mathcal{E}$ Neglect, 31, 871-883.

Correctional Service Canada. (8 de junio de 2008). Female sex offenders in the Correctional Service of Canada, case studies. Recuperado de http://www. csc-scc.gc.ca/text/pblct/so/female/female-02-eng. shtml

Denov, M. (2002). To a safer place? Victims of sexual abuse by females and their disclosures to professionals. Child Abuse Eु Neglect, 27, 47-61.

Gannon, T., \& Rose, M. (2008). Female child sexual offenders: Towards integrating theory and practice. Aggression and Violent Behavior, 13(6), 442-461.

Finkelhor, D. \& Russell, D. (1984). Women as perpetrators: Review of the evidence. En D. Finkelhor, Child Sexual Abuse: New Theory and Research (pp. 171-187). New York, Estados Unidos: Free Press.

González, E., Martínez V., Leyton, C., \& Bardi, A. (2004). Características de los abusadores sexuales. Revista SOGIA, 11(1), 6-14.

Johansson-Love, J., \& Fremouw, W. (2006). A critique of the female sexual perpetrator research. Aggression and Violent Behavior, 11(1), 12-26.

Johansson-Love, J., \& Fremouw, W. (2009). Female sex offenders: A controlled comparison of offender and victim/crime characteristics. Journal of Family Violence, 24(6), 367-376.

Kaufman, K., Wallace, A., Johnson, C. \& Reeder, M. (1995) Comparing Female and Male Perpetrators' Modus Operandi: Victims' Reports of Sexual Abuse. Journal of Interpersonal Violence,10, 322 333.

Kelly, R., Wood, J., Gonzalez, L., MacDonald, V., \& Waterman, J. (2002). Effects of mother-son incest and positive perceptions of sexual abuse experiences on the psychosocial adjustment of clinic-referred men. Child Abuse EB Neglect, 26, 425-441.

Noemí, M. (2004). La mujer en la teoría criminológica. La Ventana, 20, 240-266.

Onetto, I., \& Araya, P. (2007). Agresoras sexuales femeninas: evaluación retrospectiva de 58 mujeres imputa- 
das y evaluadas por este delito en el servicio médico legal de Chile en un periodo de 6 años. Santiago: Centro de Estudios de Justicia de las Américas. Recuperado de http://www.cejamericas.org/portal/ index.php/es/

biblioteca/bibliotecavirtual/doc_details/2210-agresorassexuales-femeninas

Pereda, N. (2006). Malestar psicológico en estudiantes universitarios víctimas de abuso sexual infantil y otros estresores (Tesis Doctoral). Universitat de Barcelona, Barcelona, España.

Robertiello, G., \& Terry, K. (2007). Can we profile sex offenders? A review of sex offender typologies. Aggression and Violent Behavior, 12(5), 508-518.

Romero, M. (2003). ¿Por qué delinquen las mujeres? Parte II. Vertientes analíticas desde una perspectiva de género. Salud Mental, 26(1), 32-41.
Sandler, J., \& Freeman, N. (2007). Typology of female sex offenders: A test of Vandiver and Kercher. Sexual Abuse: A Journal of Research and Treatment, 19, 73-89

Salinas, M. (2006). Contextos de ocurrencia de la victimización sexual: relevancia diagnóstica. Opúsculos de Derecho Penal y Criminología (№ 97). Córdoba, AR: Editorial Lerner.

Strickland, S. (2008) Female Sex Offenders Exploring Issues of Personality, Trauma, and Cognitive Distortions. Journal of Interpersonal Violence, 23(4), 474-489.

Tardif, M., Auclair, N., Jacob, M., \& Carpentier, J. (2005). Sexual abuse perpetrated by adult and juvenile females: An ultimate attempt to resolve a conflict associated with maternal identity. Child Abuse $\mathbb{E}$ Neglect, 29, 153-167. 
